\title{
A family based whole exome sequence study to indentify modifier genes for phenotype heterogeneity between severe and non-severe thalassemia patients
}

\author{
Dipankar Saha ${ }^{a}$, Prosanto Kumar Chowdhury a, Debashis Pala, Kaustav Nayek ${ }^{d}$, Gispati Chakrabortye, \\ Surupa Basub, Anupam Basua*
}

a Department of Zoology, The University of Burdwan, PurboBarddhaman- 713104, West Bengal, India. b Institute of Child Health, Kolkata, c Thalassemia Control Unit, Burdwan Medical College and Hospital, department of Paediatric Medicine, Burdwan Medical College and Hospital, ${ }^{e}$ Burdwan University Health Centre, The University of Burdwan,

\begin{abstract}
Whole Expanded Exome Sequencing Study of Keywords: WES; Thalassemia; phenotype two families father, mother and index cases (trio) was undertaken for two E/ beta Introduction:

thalassemia subjects with same HBB Thalassemia is a monogenic disease caused genotype. Approximately 200ng of DNA was by the mutation in the beta globin (HBB) gene. taken from each individual and shared into There are more than 400 disease causing $300-400 \mathrm{bp}$ fragment. Then the shared mutation is responsible for the causing beta fragments are end repair. Klenow thalassemia worldwide. (hbvar ref). HbE beta exonuclease was used to add an adapter. thalassemia is a compound heterozygous After adapter ligation 10 cycle PCR mutation of the HBB gene, found in the South amplification was done for each sample. The Esat Asia. In Eastern India there are almost targeted Exome was captured by the Agilent $17 \mathrm{HBB}$ mutation are responsible for the Sure select XT Human all Exome V6+ UTR kit disease. The most common mutations are IVS as per the manufacturer's protocol. Captured 1-5(G>C) [HBB:c.92+5G>C] and CD26(G>A) library was then amplified 10 cycles with 8 bp [HBB:c.79G>A], responsible for the HbE- beta index sequence for each sample. Then the thalassemia (1). The clinical severity of the indexed capture library was pooled together. HbE-beta thalassemia is varying from very Pair end sequencing of the pooled library was severe to very mild. Some subjects are performed in Illumina HiSeq2500 using presenting the disease at very early age and Illumina HiSeq SBS kit. Finally, both the needing regular transfusion for survival, but genes, inherited and denovo, from both the some are presenting late onset and not subjects were separately functionally requiring regular transfusion or required annotated by DAVID online tools 6.8. occasional transfusion $(2,3)$.Several studies Functionally annotation result shows that in are done to find out the factors responsible for case of subject-1, $6 \mathrm{KEGG}$ pathway were the clinical severity of the HbE-beta involved. These are Adherent junction, Protein thalassemia subjects. Several studies are digestion and absorption, Inflamatory Bowel showing the modifiers like beta mutation type, Disease, Amoebiasis, PPAR signaling fetal hemoglobin level, genetic polymorphism, pathway and glycolysis or gluconeogenesis. like -158(C>T) on Gy globin gene(4), HBS1LInterestingly in case of subject-2, only 2 MYB intergenic polymorphism, BCL11A gene KEGG pathway were found, Thyroid hormone polymorphism, $(5,6,7)$ responsible for the $\mathrm{HbF}$ synthesis and carbon metabolism. inducer and also the alpha globin gene deletions. The result shows some are
\end{abstract}

* Corresponding author: Prof. Anupam Basu, Department of Zoology, The University of Burdwan, PurboBarddhaman, West Bengal- 713104, India, Email- abasu@zoo.buruniv.ac.in 
statistically significant, but this is not enough Approximately 200ng of DNA was taken from to understand the reason of such clinical each individual and shared into $300-400 \mathrm{bp}$ diversity. So, we hypothesized that there are fragment. Then the shared fragments are end several other genetic loci, other than the repair. Klenow exonuclease was used to add globin cluster are present, which may modify an adapter. After adapter ligation 10 cycle the disease severity in the different way other PCR amplification was done for each sample. than the modulation of the globin production.

To finding out the genetic modifiers, responsible for the disease severity of the HbE-Beta thalassemia subject, with similar genotype [IVS1-5(G>C)/CD16(G>A)] we did Whole Expanded Exom Sequencing Study of two family (father, mother and affected patients).

\section{Materials and Methods:}

The targeted Exome was captured by the Agilent Sure select XT Human all Exome V6+ UTR kit as per the manufacturer's protocol. Captured library was then amplified 10 cycles with $8 \mathrm{bp}$ index sequence for each sample. Then the indexed capture library was pooled together. Pair end sequencing of the pooled library was performed in Illumina HiSeq2500 using Illumina HiSeq SBS kit V4 as per manufacturer's protocol. Generated FASTQ file was used for the variant calling and in house data analysis.

Subjects Information: We select two HbEBeta thalassemia subjects with same HBB genotype IVS1-5(G>C) and CD26(G>A) with different phenotype: One is transfusion dependent (TD) and another one is non transfusion dependent (NTD). To check whether any de novo mutations have any role as modifier loci, parents of the both the subjects were also included in this study. Clinicopathological information of both the subjects have been presented table-1.

Subject-1, which is TDT present the disease at 10 month of age and taking regular transfusion of 2-month interval for last 13 year. Steady state haemoglobin level was 6.1 $\mathrm{g} / \mathrm{dl}$. Subject- 2, which is NTDT present the disease at 14 years of age. She has not taking any transfusion for last 16 years. Steady state haemoglobin was $7.8 \mathrm{~g} / \mathrm{dl}$. She has a
splenomegaly of $9 \mathrm{~cm}$ from the left costal margin.

A total of 6 subjects were included in this study as described above including both the parents of each index cases. The study has been approved by the Institutional Cinical Ethics Committee, The university of Burdwan

Sample Collection and DNA extraction: Pre transfused $3 \mathrm{ml}$ peripheral blood samples were collected from individual subjects in EDTA from each subjects with proper phlebotomy procedure. DNA was extracted by using the commercial DNA extraction kit.

Whole Exome sequencing:

WES Data Analysis:

Variant calling: All the raw reads were checked and aligned with hg19 human reference genome using Burrows- Wheeler Aligner (BWA) and variant call was made by Genomic Analysis Tool Kit (GATK). The VCF was annotated by using the VarAFT tools.

\section{Hunting of responsible variants and gene for clinical significance:}

De novo variants: Variants of the Index cases were compared with the variants of the father and mother and inherited variants were filtered out.

Inherited variants: After annotating the VCF file using VarAFT tools we filter out all Upstream, downstream, intergenic, UTR, nocoding and non splicing related intronic variants, only exonic and splicing variants are considered for further analysis. To hunt the responsible Inherited variants two broad scheme were applied. In the first scheme, only minor variants were targeted. In the second scheme, homozygous pathogenic variants were targeted as follows:

Scheme 1: We considered only nonsynonymous exonic variant and splicing variants with frequency of $<0.01(1 \%)$ in 1000 Genome database, for the next step of analysis. We align the individual's variants with the father, mother variants to detect the inherited and de novo variants. The variants were again filter based on the SIFT and PolyPhen2 score. The variants showing 
pathogenic either any of one were taken for the final variants. Then the variants were maped into gene and the genes were functionally annotated with the DAVID online tools 6.8 to searching out the biological pathway, KEGG pathway involved with these genes.

Scheme 2: All the synonymous, unknown and heterozygous variants are further filtrated. To predict the effect of mutation, SIFT and POLYPHEN2 scoring were applied on all the homozygous exonic and splice variants only deleterious variants by this two software were considered. The genes containing these variants are further functionally clustered by KEGG path way using DAVID online tools 6.8.

\section{Results}

A total of 91194 variants were identified in the TDT subject and 91196,90530 variants were identified in the father and mother respectively. On the other hand a total of 90226 variants were identified in the NTDT subject and 89396,88275 variants were identified in the father and mother respectively.

\section{De novo Variants}

After filtering out the finally we got the 520, 551 and 503 non synonymous exonic and splicing variants in subject-1, father and mother respectively. Out of 520 variants, 514 are inherited variants and only 6 are denovo variants for TDT subject. On the other hand, 538,530 and 541 variants were found in NTDT subject, father and mother respectively. Out of 538 total variants in index case, 534 were inherited variants and 4 were de novo variants (Table 2 ) .

\section{Inherited variants and functional annotation:}

As mentioned in the method section, for hunting of the functional clinical responsible variants and genes, using minor allele approach (Fig 1) and homozygous variants approach (Fig 2) available genes and variants are listed in Table $3-6$.

\section{Discussion}

We performed a trio based study with 2 beta haemoglobinopathy patients of same age, sex, and harbouring the same primary mutation, however, one was transfusiondependent (TDT) and the other nontransfusion-dependent (NTDT). We focussed on single-nucleotide variations and short indels in a whole-exome sequencing approach, and then tried to explain their differing clinical phenotypes through their differing mutations with expected most severe impact. After associating mutations of gene products to the metabolic pathways they have most significant roles in, we found that the mutations and their related individual phenotypes belonged to either of 2 categories - those related to general defects of growth and development, and those related to anaemia, either by reduced/ineffective erythropoiesis or by increased RBC haemolysis.

In our cases of interest, we found that mutations of genes associated with growth and development were present in both patients, while those possibly involved in erythrocyte membrane or even general cytoskeletal integrity were affected in only the TDT individual. Also interesting was that the NTDT patient displayed a much lower mutational load than the TDT patient (about $1 / 3^{\text {rd }}$ of the TDT patient's mutational load).

Patient 1 (addressed as the TDT patient), was diagnosed at 10 months of age and was on once in 2-3 months transfusion regimen from then. She was maintaining and average pre transfusion haemoglobin level of $6.05 \mathrm{gm} / \mathrm{dl}$. Her height and weight for age were both below the $3^{\text {rd }}$ centile against age and sex matched subjects. Both the liver and spleen were palpably enlarged to the extent of 4.5 and $7 \mathrm{~cm}$ below the right and left costal margins respectively. The level of lactate Dehydrogenase, serum haptoglobin and unconjugated bilirubin remained significantly above normal range, during this period (8). Provisionally attributing the increase of such parameters to excessive ineffective erythropoiesis she was put on regular monthly transfusion from 7 years of age. Attempting to 
maintain average pre-transfusion is known to be dysregulated in sickling RBCs haemoglobin level of above $9 \mathrm{gm} / \mathrm{dl}$ with 15 (11). These mutations could explain the extra $\mathrm{ml} / \mathrm{kg}$ of $60 \%$ packed cell transfusion. Though burden of ineffective erythropoiesis and the biochemical parameters of ineffective intravascular haemolysis, evidenced by erythropoiesis improved but never touched baseline, this hinting at intravascular haemolysis as a probable cause (9). Upon starting on the new transfusion regime, minimal improvement in growth was noted. Though the average pretransfusion haemoglobin level remained around $8 \mathrm{gm} / \mathrm{dl}$, with 3 weeks transfusion interval regime. The iron overload increased at a rate more than expected. After follow up till 18 years of age, it was noted that she was having a number of comorbidities, not unusual for thalassaemics but at an earlier age. She was detected as bio-chemically hypothyroid with free triiodothyronine level of $0.8 \mathrm{ng} / \mathrm{ml}$ and Thyroid stimulating hormone level of $7.02 \mathrm{ng} / \mathrm{ml}$, which was corrected with levothyroxine replacement therapy. She had thelarche at 16 years and menarche at 18 years of age, both delayed by about 2 years, following the secular trend. The bone age at 18 years was lagging by 2 years and the $\mathrm{Z}$ Score of bone mineral density at trochanteric level was -1.0, signifying Osteopenia. She was detected as having multiple small gall bladder stones at the same age. We also contributed this delay of catch up growth and development to a chronic allergic state and chronic ill health due frequent abdominal cramps and diarrhoea leading to malabsorption and micronutrient deficiency. After replacement therapy with essential mineral (calcium and zinc) and vitamin (Vit $D$ ) replacement and probiotics, her condition has improved, but her growth still remains below the $3^{\text {rd }}$ centile with moderately enlarged liver and spleen.

After the data of the NGS was made available, it was noted that she was compound heterozygous for an ACTN3 mutation, which is thought to be involved in attachment of actin filaments to intracellular structures. This mutation could have far-reaching consequences, though in RBC it would most likely lead to structural destabilization and increased erythrolysis, thus exacerbating the anaemic condition and enforcing a transfusion-dependent state (10). Also present was a mutation in FARP2, a guanine exchange factor involved in cytoskeletal remodelling by RAC1, which has been implicated in erythrocyte maturation (12) and hyperbilirubinemia, high LDH and Haptoglobin and compensatory increase in size of liver and spleen and formation of gall stones at such an early age. Outside of anaemic conditions, though, there were mutations identified in both patients relating to various pathways of growth and development, with a common motif being genes involved in cellular respiration and fatty acid metabolism. Outside of genes involved in carbon metabolism, the TDT subject possessed mutations in IL12RB1 and TLR4, which may be contributory to defective $T$ Helper cell-mediated immune response and gamma interferon production and its related antiviral response (15) - the propensity of this subject to fall sick very often, mostly to influenza like viral illness, wrongly interpreted as allergic hyperactive airway disorder (14, 15). More over IL12RB1 mutation may be responsible for inflammatory bowel disease like state $(13,16)$, which could be responsible for the malabsorption like symptoms leading to lactose intolerance and micronutrient deficiency. Reduced bone mineral density and resultant osteopenia may, among other things, be attributable to the menagerie of collagen deficiencies. (17).

Learning that this patient also presented with very early inset hypothyroidism, which is unlikely in well chelated thalassaemic individuals association with homozygous mutations in the HLA-DR3, HLA-DPB1 and the TPO genes may be corroborative. It has been shown that individuals with an amino acid substitution at position 74 of the DR beta 1 chain of HLA-DR3 (DRb1-Arg74), susceptibility to autoimmune thyroid disorder increases [18]. The analysis of amino acid variants of HLA molecules of HLA-DPB1 were strongly associated with Graves Disease, especially amino-acid signatures of the HLADP $\beta$ chain, might contribute to the molecular pathogenesis of early-onset auto-immune thyroid disease (AITD) [19]. TPO gene mutations result in disruption of thyroid hormone synthesis and are classified as thyroid dyshormonogenesis. The combined effects of these genetic aberrations may have contributed to the individual presenting as clinically and biochemically hypothyroid at such an early age, to require thyroid hormone replacement therapy. [20] Presence of homozygous mutation in the FUT3 gene may be of significance in relation to increased 
transfusion frequency. Mutation in FUT3 gene contributing defects in the RBC cytoskeleton, is actually reflected as the gene for Lewis when compared to the TDT subject.

Antigen $\left(\mathrm{Le}^{\mathrm{a}+}\right.$ or $\left.\mathrm{Le}^{\mathrm{b}+}\right)$ a minor blood group antigen, so the person who will not be able to synthesize the antigen (protein product of the Le gene) and will be Le Negative $\left(\mathrm{Le}^{\mathrm{a}-} \mathrm{Le}^{\mathrm{b}-}\right)$, as the transfusable blood comprising of th packed red cells are generally not screened for such minor blood groups, to in individuals who receive frequent transfusions, may develop Anti Lewis antibodies to such seemingly innocuous antigen, thus causing partial haemolysis of the transfused blood which is positive for such antigen. This small amount of haemolysis is not severe enough to cause catastrophic transfusion reaction, but will decrease the transfusion interval subtly, increasing the levels of bilirubin and LDH. This could be another reason for increased haemolysis which persisted even after starting the regular transfusions, which should have ideally got rid of the endogenously produced defective red cells. Generally, in sporadically transfused individuals Lewis blood group is seldom responsible for such haemolysis, however in multi and regular transfusion scenarios, it compromises transfusion efficiency, hence decreasing the frequency between transfusions. [21] Though the mutations in the FUT3 may be associated with $\mathrm{H}$. Pylori infection causing frequent abdominal cramps, etc, this has not been substantiated in our subject. [Ref Table 4]

Apart from a considerably larger mutational load on fatty acid metabolic pathway genes in the TDT patient, thyroid hormone production was almost equally affected in both. Other than this pathway in the NTDT patient, angiogenesis, limb and connective tissue (collagen) formation and immune development were severely affected in the TDT individual, sometimes even multifactorial.

When, we compared the clinical condition of Index case 2, the NTDT, it was certainly different. She presented at the clinical set up at the age of 12 years, with and average baseline haemoglobin of $6.0 \mathrm{gm} / \mathrm{dl}$, with no hepatomegaly and palpable splenomegaly of $6 \mathrm{~cm}$ below the left costal margin. The LDH, Authors declare there is no conflict of interest unconjugated bilirubin and haptoglobin were in the present study.

only more than 2xupper limit of normal (ULN), rest of the parameters being normal and documenting only $20 \%$ Nucleated red cells in The authors are grateful to Department of peripheral circulation. These findings are truly Biotechnology, Govt of India for funding this correlated with the absence of mutations work [ Sanc. No- 
also express due acknowledgment to National Institute of Biomedical Genomics (NIBMG), Kalyani for providing necessary out sourced service for WES work]

\section{References}

1. Panja A, Chowdhury P, Chakraborty $S$, Ghosh TK, Basu A. Cross-Sectional Study for the Detection of Mutations in the Beta-Globin Gene Among Patients with Hemoglobinopathies in the Bengali Population. Genet Test $\mathrm{Mol}$ Biomarkers. 2017;21(1):39-45. doi:10.1089/gtmb.2016.0186

2. Setianingsih I, Williamson R, Daud D, Harahap A, Marzuki S, Forrest S. Phenotypic variability of Filipino beta(o)-thalassemia/ $\mathrm{HbE}$ patients in Indonesia. Am J Hematol. 1999;62(1):7-12.

doi:10.1002/(sici)1096-

8652(199909)62:1<7::aid-

ajh2>3.0.co;2-s

3. Sharma V, Kumar B, Kumar G, Saxena R. Alpha globin gene numbers: an important modifier of $\mathrm{HbE} /$ beta thalassemia. Hematology. 2009;14(5):297-300.

doi:10.1179/102453309X446126

4. Charoenkwan $P$, Teerachaimahit $P$, Sanguansermsri $T$. The correlation of a-globin gene mutations and the $\mathrm{Xmnl}$ polymorphism with clinical severity of $\mathrm{Hb} \quad E / \beta$-thalassemia. Hemoglobin. 2014;38(5):335-338. doi:10.3109/03630269.2014.952744

5. Bandyopadhyay S, Roychowdhury K, Chandra S, Das M, Dasgupta UB. Variable severity of beta-thalassemia patients of eastern India: effect of alpha-thalassemia and $\mathrm{xmnl}$ polymorphism. Clin Exp Med. 2001;1(3):155-159. doi:10.1007/s10238-001-8028-x

6. Phanrahan $P$, Yamsri S, Teawtrakul N, Fucharoen G, Sanchaisuriya K, Fucharoen S. Molecular Analysis of Non-Transfusion

Dependent Thalassemia Associated with
Hemoglobin E- $\beta$-Thalassemia Disease without $\alpha$-Thalassemia. Mediterr $J$ Hematol Infect Dis. 2019;11(1):e2019038. Published 2019 Jul 1. doi:10.4084/MJHID.2019.038

7. Ray R, Kalantri SA, Bhattacharjee S, et al. Association of alpha hemoglobinstabilizing protein (AHSP) gene mutation and disease severity among HbE-beta thalassemia patients. Ann Hematol. 2019;98(8):1827-1834. doi:10.1007/s00277-019-03722-x

8. Oikonomidou, Paraskevi Rea, and Stefano Rivella. "What can we learn from ineffective erythropoiesis in thalassemia?." Blood reviews 32.2 (2018): 130-143.

9. Conran, Nicola. "Intravascular hemolysis: a disease mechanism not to be ignored." Acta Haematol 132.1 (2014): 97-99.

10. Gokhin, David S., and Velia M. Fowler. "Feisty filaments: actin dynamics in the red blood cell membrane skeleton." Current opinion in hematology 23.3 (2016): 206.

11. George, Alex, et al. "Altered phosphorylation of cytoskeleton proteins in sickle red blood cells: the role of protein kinase C, Rac GTPases, and reactive oxygen species." Blood Cells, Molecules, and Diseases 45.1 (2010): 41-45.

12. Konstantinidis, Diamantis G., et al. "Signaling and cytoskeletal requirements in erythroblast enucleation." Blood, The Journal of the American Society of Hematology 119.25 (2012): 61186127.

13. Lu, Yue, et al. "Toll-like receptors and inflammatory bowel disease." Frontiers in immunology 9 (2018): 72.

14. Monteiro, Juanita M., Catherine Harvey, and Giorgio Trinchieri. "Role of interleukin-12 in primary influenza virus infection." Journal of virology 72.6 (1998): 4825-4831.

15. Guo, Yifei, Wei Cao, and Ying Zhu. "Immunoregulatory Functions of the IL12 Family of Cytokines in Antiviral Systems." Viruses 11.9 (2019): 772.

16. Cao, Anthony T., et al. "TLR4 regulates IFN- $\mathrm{Y}$ and $\mathrm{IL}-17$ production 
by both thymic and induced Foxp3+ Tregs during intestinal inflammation." Journal of leukocyte biology 96.5 (2014): 895-905.

17. Xie, Peigen, et al. "Association of COL1A1 polymorphisms with osteoporosis: a meta-analysis of clinical studies." International journal of clinical and experimental medicine 8.9 (2015): 14764.

18. Jacobson, Eric M., Amanda Huber, and Yaron Tomer. "The HLA gene complex in thyroid autoimmunity: from epidemiology to etiology." Journal of autoimmunity 30.1-2 (2008): 58-62.
19. Shin, Dong-Hwan, et al. "HLA alleles, especially amino-acid signatures of HLA-DPB1, might contribute to the molecular pathogenesis of early-onset autoimmune thyroid disease." PloS one 14.5 (2019): e0216941.

20. Cangul, Hakan, et al. "Thyroid dyshormonogenesis is mainly caused by TPO mutations in consanguineous community." Clinical endocrinology 79.2 (2013): 275-281.

21. Peng and Meunier. Lewis System: Anti Lewis, Canadian Blood Services. Professional Education. Oct 2019 
Table-1. Clinical findings of two subjects:

\begin{tabular}{|l|l|l|}
\hline Clinical findings & Severe & Non severe \\
\hline Present age & 13 year & 16 year \\
\hline Sex & Female & Female \\
\hline Age of onset & 10 month & 14 year \\
\hline Baseline haemoglobin & 6.1 gm/dl & 7.8 gm/dl \\
\hline Transfusion Interval & 2 month & No transfusion yet \\
\hline Spleen size & $5 \mathrm{~cm}$ & $9 \mathrm{~cm}$ \\
\hline MCV (fl) & 61.1 & 64.4 \\
\hline MCH (pg) & 18.1 & 19.3 \\
\hline RDW\% & 28.5 & 29.8 \\
\hline HPLC impression & HbE- $\beta$ thalassemia & HbE- $\beta$ thalassemia \\
\hline HbAO (\%) & 3.1 & 6.6 \\
\hline HbF (\%) & 50.3 & 31.5 \\
\hline HbA2+E (\%) & 42.6 & 57.4 \\
\hline
\end{tabular}


Table 2: Dene novo variants of both TDT and NTDT subjects

\begin{tabular}{|l|l|l|l|l|l|l|l|}
\hline Subject & Gene & db SNP ID & Chr & Ref & Alt & Func. refgene & Genotype \\
\hline \multirow{5}{*}{ TDT } & SPDYE1 & rs573518672 & chr7 & AGG & - & splicing & het \\
\cline { 2 - 8 } & NBPF15 & rs200795677 & chr1 & G & A & exonic & het \\
\cline { 2 - 8 } & BEX4 & rs775129946 & chrX & A & G & exonic & het \\
\cline { 2 - 8 } & SYNE2 & rs546650178 & chr14 & C & T & exonic & het \\
\cline { 2 - 8 } & KCNH1 & rs572710174 & chr1 & AAA & - & splicing & hom \\
\cline { 2 - 8 } & OR2A7 & rs200085936 & chr7 & G & C & exonic & het \\
\hline
\end{tabular}


Table 3: Involvement of the responsible alter Genes involved in different metabolic /or molecular pathway and their variants details for TDT as obtained through scheme 1 for minor allele hunting .

\begin{tabular}{|c|c|c|c|c|c|c|c|}
\hline Pathway & Gene & db SNP ID & Chr & Ref & Alt & Func.refgene & Genotype \\
\hline \multirow{6}{*}{$\begin{array}{l}\text { Adherens } \\
\text { junction }\end{array}$} & FARP2 & rs145392931 & chr2 & $\mathrm{C}$ & $\mathrm{T}$ & splicing & het \\
\hline & LMO7 & rs199685242 & chr13 & G & $\mathrm{T}$ & exonic & het \\
\hline & ACTN3 & rs200452235 & chr11 & G & $A$ & exonic;splicing & het \\
\hline & ACTN3 & rs201487054 & chr11 & G & A & exonic & het \\
\hline & FGFR1 & rs542417198 & chr8 & A & G & splicing & het \\
\hline & PTPRB & rs112571541 & chr12 & A & $\mathrm{T}$ & splicing & het \\
\hline \multirow{5}{*}{$\begin{array}{l}\text { Protein } \\
\text { digestion }\end{array}$} & COL1A1 & rs66592376 & chr17 & G & $\mathrm{T}$ & splicing & het \\
\hline & COL6A1 & rs200261890 & chr21 & G & A & exonic & het \\
\hline & COL6A3 & rs146546544 & chr2 & G & A & exonic & het \\
\hline & COL6A3 & rs111228504 & chr2 & G & $\mathrm{A}$ & splicing & het \\
\hline & COL21A1 & rs528003403 & chr6 & $A$ & G & splicing & het \\
\hline \multirow[b]{4}{*}{ IBD } & RORA & rs200399670 & chr15 & A & $\mathrm{T}$ & exonic;splicing & het \\
\hline & IL12RB1 & rs369576406 & chr19 & $\mathrm{C}$ & $\mathrm{T}$ & exonic & het \\
\hline & HLA-DOA & rs144931749 & chr6 & $\mathrm{C}$ & $\mathrm{T}$ & exonic & het \\
\hline & TLR4 & rs137853920 & chr9 & G & $\mathrm{A}$ & exonic & het \\
\hline \multirow[b]{6}{*}{ Amoebiasis } & ACTN3 & rs200452235 & chr11 & G & A & exonic;splicing & het \\
\hline & ACTN3 & rs201487054 & chr11 & G & A & exonic & het \\
\hline & COL1A1 & rs66592376 & chr17 & G & $\mathrm{T}$ & splicing & het \\
\hline & $\mathrm{C9}$ & rs121909592 & chr5 & G & A & exonic & het \\
\hline & MUC2 & rs182669692 & chr11 & $\mathrm{G}$ & $A$ & exonic & het \\
\hline & TLR4 & rs137853920 & chr9 & G & A & exonic & het \\
\hline \multirow[b]{4}{*}{ Glycolysis } & $\mathrm{ADH} 1 \mathrm{C}$ & rs6413444 & chr4 & G & A & exonic & het \\
\hline & FBP1 & rs200948424 & chr9 & $\mathrm{C}$ & G & splicing & het \\
\hline & PCK2 & rs201974284 & chr14 & G & $A$ & exonic & het \\
\hline & PGK2 & rs140642109 & chr6 & $\mathrm{T}$ & A & exonic & het \\
\hline \multirow{4}{*}{$\begin{array}{l}\text { PPAR } \\
\text { signalling }\end{array}$} & CPT1A & rs374383052 & chr11 & $\mathrm{C}$ & $\mathrm{T}$ & exonic & het \\
\hline & PLIN1 & rs554749197 & chr15 & $\mathrm{T}$ & $\mathrm{C}$ & exonic & het \\
\hline & PPARA & rs571930753 & chr22 & A & C & exonic & het \\
\hline & PCK2 & rs201974284 & chr14 & G & A & exonic & het \\
\hline
\end{tabular}


Table 4. Involvement of the responsible alter Genes involved in different metabolic /or molecular pathway and their variants details for TDT as obtained through scheme 2 for homozygous pathogenic loci hunting

\begin{tabular}{|c|c|c|c|c|c|c|c|}
\hline Pathway & Gene & db SNP ID & Chr & Ref & Alt & Func. refgene & Genotype \\
\hline \multirow[b]{10}{*}{$\begin{array}{l}\text { Auto immune } \\
\text { thyroid diseases }\end{array}$} & HLA-A & rs199474424 & 6 & $\mathrm{G}$ & $\mathrm{C}$ & exonic & Homozygous \\
\hline & HLA-A & rs2230991 & 6 & $\mathrm{G}$ & $A$ & exonic & Homozygous \\
\hline & HLA-A & rs3173420 & 6 & G & $A$ & exonic & Homozygous \\
\hline & HLA-B & rs1050723 & chr6 & G & $A$ & exonic & hom \\
\hline & HLA-B & rs1050570 & chr6 & $\mathrm{T}$ & $\mathrm{C}$ & exonic & hom \\
\hline & HLA-B & rs1065386 & chr6 & $\mathrm{G}$ & C & exonic & hom \\
\hline & HLA-B & rs1050529 & chr6 & $\mathrm{C}$ & $T$ & exonic & hom \\
\hline & HLA-C & rs41542414 & chr6 & $A$ & $T$ & exonic & hom \\
\hline & $\begin{array}{l}\text { HLA- } \\
\text { DPB1 }\end{array}$ & rs1042153 & chr6 & $\mathrm{G}$ & $A$ & exonic & hom \\
\hline & TPO & rs2175977 & chr2 & $\mathrm{G}$ & $\mathrm{C}$ & exonic & hom \\
\hline \multirow{9}{*}{$\begin{array}{l}\text { Graft-versus-host } \\
\text { disease/ Allograft } \\
\text { rejection } \\
\text { and } \\
\text { Type I diabetes } \\
\text { mellitus }\end{array}$} & HLA-A & rs199474424 & chr6 & $\mathrm{G}$ & $\mathrm{C}$ & exonic & hom \\
\hline & HLA-A & rs2230991 & chr6 & $\mathrm{G}$ & $A$ & exonic & hom \\
\hline & HLA-A & rs3173420 & chr6 & $\mathrm{G}$ & $A$ & exonic & hom \\
\hline & HLA-B & rs1050723 & chr6 & $\mathrm{G}$ & $A$ & exonic & hom \\
\hline & HLA-B & rs1050570 & chr6 & $T$ & C & exonic & hom \\
\hline & HLA-B & rs1065386 & chr6 & $\mathrm{G}$ & $\mathrm{C}$ & exonic & hom \\
\hline & HLA-B & rs1050529 & chr6 & $\mathrm{C}$ & $T$ & exonic & hom \\
\hline & HLA-C & rs41542414 & chr6 & $A$ & $T$ & exonic & hom \\
\hline & $\begin{array}{l}\text { HLA- } \\
\text { DPB1 }\end{array}$ & rs1042153 & chr6 & G & A & exonic & hom \\
\hline \multirow{3}{*}{$\begin{array}{l}\text { Glycosphingolipid } \\
\text { biosynthesis }\end{array}$} & FUT3 & rs778986 & chr19 & $A$ & G & exonic & hom \\
\hline & FUT5 & rs4807054 & chr19 & G & $A$ & exonic & hom \\
\hline & FUT9 & rs3811069 & chr6 & $A$ & G & exonic & hom \\
\hline \multirow[b]{6}{*}{$\begin{array}{l}\text { ECM-receptor } \\
\text { interaction }\end{array}$} & COL6A6 & rs9830253 & chr3 & G & $A$ & exonic & hom \\
\hline & ITGA11 & rs4777035 & chr15 & G & $A$ & exonic & hom \\
\hline & LAMA5 & rs944895 & chr20 & $\mathrm{G}$ & $A$ & exonic;splicing & hom \\
\hline & LAMA5 & rs2427283 & chr20 & $\mathrm{C}$ & $T$ & exonic & hom \\
\hline & TNN & rs2072036 & chr1 & C & $T$ & exonic & hom \\
\hline & VTN & rs704 & chr17 & G & $A$ & exonic & hom \\
\hline
\end{tabular}


Table 5: Involvement of the responsible alter Genes involved in different metabolic /or molecular pathway and their variants details for NTDT as obtained through scheme 1 for minor allele hunting .

\begin{tabular}{|c|c|c|c|c|c|c|c|}
\hline $\begin{array}{l}\text { KEGG } \\
\text { pathway }\end{array}$ & Gene & db SNP ID & Chr & Ref & Alt & Func.refgene & Gen \\
\hline \multirow{4}{*}{$\begin{array}{l}\text { Thyroid } \\
\text { hormone } \\
\text { synthesis }\end{array}$} & $\mathrm{TG}$ & rs35301433 & chr8 & $\mathrm{A}$ & $\mathrm{G}$ & exonic & het \\
\hline & SLC26A4 & rs375576481 & chr7 & A & $\mathrm{T}$ & exonic & het \\
\hline & LRP2 & rs41268685 & $\operatorname{chr} 2$ & $\mathrm{C}$ & $\mathrm{T}$ & exonic & het \\
\hline & GPX6 & rs562827573 & $\operatorname{chr} 6$ & $\mathrm{C}$ & $\mathrm{T}$ & exonic & het \\
\hline \multirow{5}{*}{$\begin{array}{l}\text { Carbon } \\
\text { metabolism }\end{array}$} & ACO1 & rs554238047 & $\operatorname{chr} 9$ & $G$ & $\mathrm{~T}$ & exonic & het \\
\hline & G6PD & rs5030868 & $\operatorname{chrX}$ & $\mathrm{G}$ & $\mathrm{A}$ & exonic & het \\
\hline & HK1 & rs201626997 & chr10 & G & $\mathrm{T}$ & exonic;splicing & het \\
\hline & OGDHL & rs146013158 & chr10 & $\mathrm{C}$ & A & splicing & het \\
\hline & TKTL2 & rs565930006 & $\operatorname{chr} 4$ & $\mathrm{C}$ & $G$ & exonic & het \\
\hline
\end{tabular}

Table 6. Involvement of the responsible alter Genes involved in different metabolic /or molecular pathway and their variants details for NTDT as obtained through scheme 2 for homozygous pathogenic loci hunting

\begin{tabular}{|l|l|l|l|l|l|l|l|}
\hline \multirow{4}{*}{ Pathway } & Gene & db SNP ID & Chr & Ref & Alt & Func.refgene & Genotype \\
\hline \multirow{5}{*}{} & CELA3B & rs7528405 & chr1 & C & T & exonic;splicing & hom \\
\cline { 2 - 9 } & COL6A6 & rs9830253 & chr3 & G & A & exonic & hom \\
\cline { 2 - 9 } & COL6A6 & rs61629992 & chr3 & C & T & exonic & hom \\
\cline { 2 - 9 } & COL11A1 & rs3753841 & chr1 & G & A & exonic & hom \\
\cline { 2 - 9 } & COL12A1 & rs970547 & chr6 & C & T & exonic;splicing & hom \\
\cline { 2 - 8 } & COL14A1 & rs4870723 & chr8 & A & C & exonic & hom \\
$\begin{array}{l}\text { Protein } \\
\text { digestion } \\
\text { absorption }\end{array}$ & COL24A1 & rs11161732 & chr1 & G & A & exonic & hom \\
\cline { 2 - 8 }
\end{tabular}




\section{Subject-1 (TDT)}

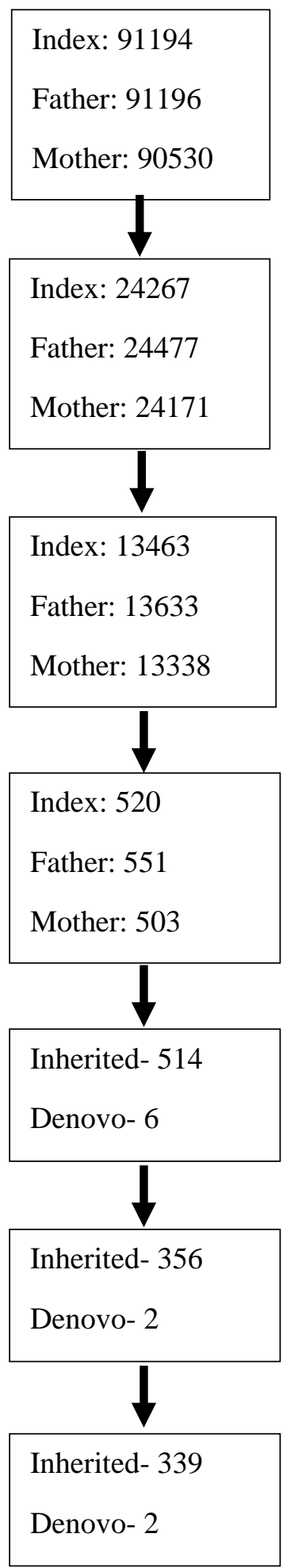

\section{Subject-2(NTDT)}

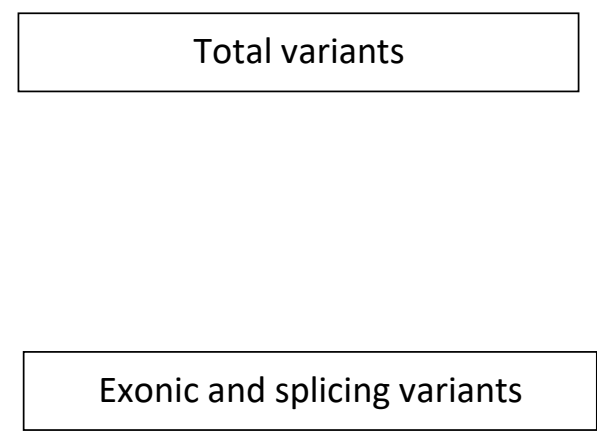

Exonic non synonymous and splicing variants

Exonic non synonymous and splicing variants frequency $<0.01$ in 1000 Genome database

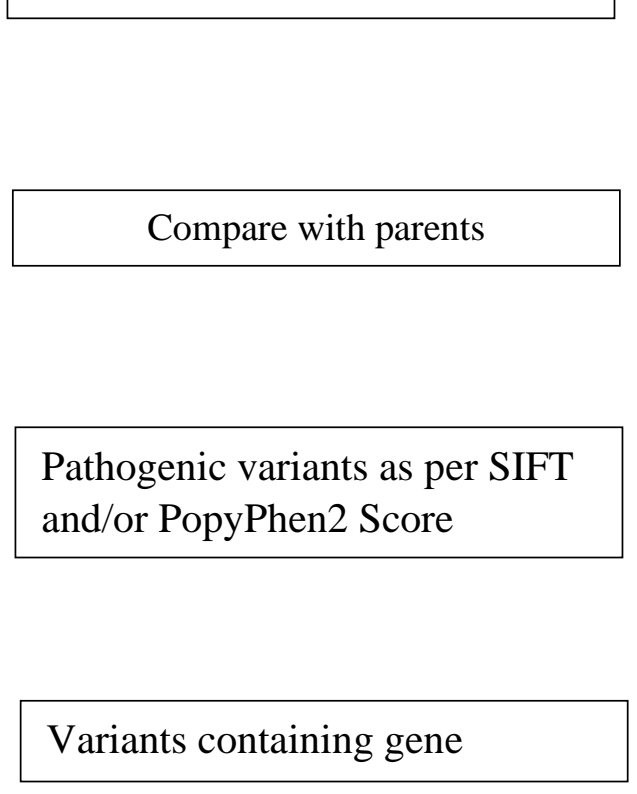

Index: 90226

Father: 89396

Mother: 88275

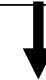

Index: 24118

Father: 23839

Mother: 23908

Index: 13398

Father: 13212

Mother: 13197

Index: 538

Father: 530

Mother: 541

Inherited- 534

Denovo- 4

Inherited- 354

Denovo- 4

Inherited- 354

Denovo- 2

Fig 1: Flowchart of the Scheme 1-for finding of the different inherited variants with minor allele frequency 


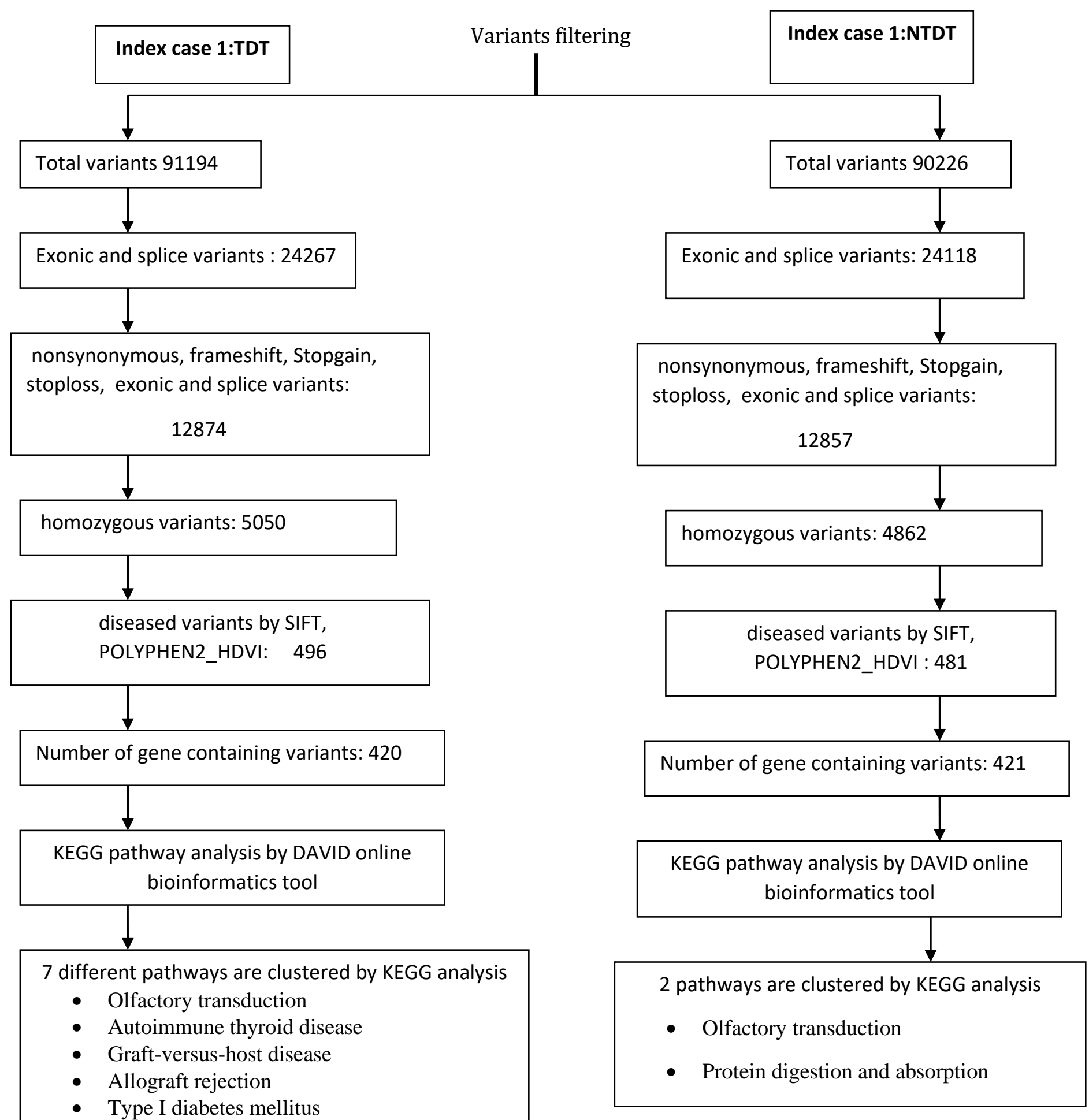

Fig 2: Flowchart of the Scheme 2-for finding of the different inherited homozygous pathogenic variants and respective gene functional clustering using KEGG analysis 


\section{Supplementary Information}

Supplementary Table 1: Functional annotation of the gene out of de novo variants of both the TDT and NTDT index subjects.

\begin{tabular}{|c|c|c|}
\hline Subject & Gene & Gene Function \\
\hline \multirow[b]{2}{*}{ TDT } & SPDYE1 & $\begin{array}{l}\text { Interacting selectively and non-covalently with a protein kinase, any enzyme } \\
\text { that catalyzes the transfer of a phosphate group, usually from ATP, to a protein } \\
\text { substrate. Diseases associated with SPDYE1 include Williams-Beuren } \\
\text { Syndrome }\end{array}$ \\
\hline & NBPF15 & $\begin{array}{l}\text { This gene is a member of the neuroblastoma breakpoint family (NBPF), } \\
\text { Members of this gene family are characterized by tandemly repeated copies of } \\
\text { DUF1220 protein domains. Diseases associated with NBPF15 } \\
\text { include Neuroblastoma and Hydrolethalus Syndrome }\end{array}$ \\
\hline \multirow{4}{*}{ NTDT } & BEX4 & $\begin{array}{l}\text { May play a role in microtubule deacetylation by negatively regulating the } \\
\text { SIRT2 deacetylase activity toward alpha-tubulin and thereby participate in the } \\
\text { control of cell cycle progression and genomic stability. The proteins encoded } \\
\text { by some of the other members of this family act as transcription elongation } \\
\text { factors which allow RNA polymerase II to escape pausing during elongation. }\end{array}$ \\
\hline & SYNE2 & $\begin{array}{l}\text { Multi-isomeric modular protein which forms a linking network between } \\
\text { organelles and the actin cytoskeleton to maintain the subcellular spatial } \\
\text { organization. As a component of the LINC (LInker of Nucleoskeleton and } \\
\text { Cytoskeleton) complex involved in the connection between the nuclear lamina } \\
\text { and the cytoskeleton. play an important role in the transmission of mechanical } \\
\text { forces across the nuclear envelope and in nuclear movement and positioning. }\end{array}$ \\
\hline & KCNH1 & $\begin{array}{l}\text { Voltage-gated potassium }(\mathrm{Kv}) \text { channels represent the most complex class of } \\
\text { voltage-gated ion channels from both functional and structural standpoints. } \\
\text { Their diverse functions include regulating neurotransmitter release, heart rate, } \\
\text { insulin secretion, neuronal excitability, epithelial electrolyte transport, smooth } \\
\text { muscle contraction, and cell volume }\end{array}$ \\
\hline & OR2A7 & $\begin{array}{l}\text { OR2A7 (Olfactory Receptor Family } 2 \text { Subfamily A Member 7) is a Protein } \\
\text { Coding gene. Among its related pathways are Signaling by } \\
\text { GPCR and Olfactory transduction. }\end{array}$ \\
\hline
\end{tabular}

